\title{
Burst pressure of phaseguide structures of different heights in all-polymer microfluidic channels
}

\author{
Garbarino, Francesca; Kistrup, Kasper; Rizzi, Giovanni; Hansen, Mikkel Fougt
}

Published in:

Journal of Micromechanics and Microengineering

Link to article, DOI:

10.1088/1361-6439/aa97b7

Publication date:

2017

Document Version

Peer reviewed version

Link back to DTU Orbit

Citation (APA):

Garbarino, F., Kistrup, K., Rizzi, G., \& Hansen, M. F. (2017). Burst pressure of phaseguide structures of different heights in all-polymer microfluidic channels. Journal of Micromechanics and Microengineering, 27(12), [125015]. https://doi.org/10.1088/1361-6439/aa97b7

\section{General rights}

Copyright and moral rights for the publications made accessible in the public portal are retained by the authors and/or other copyright owners and it is a condition of accessing publications that users recognise and abide by the legal requirements associated with these rights.

- Users may download and print one copy of any publication from the public portal for the purpose of private study or research.

- You may not further distribute the material or use it for any profit-making activity or commercial gain

- You may freely distribute the URL identifying the publication in the public portal 


\title{
Burst pressure of phaseguide structures of different heights in all-polymer microfluidic channels
}

\author{
Francesca Garbarino, Kasper Kistrup, Giovanni Rizzi and Mikkel Fougt \\ Hansen*
}

Department of Micro- and Nanotechnology, DTU Nanotech, Building 345B, Technical University of Denmark, DK-2800 Kongens Lyngby, Denmark

*E-mail: Mikkel.hansen@nanotech.dtu.dk

\begin{abstract}
We present an experimental investigation of the burst/overflow pressure of water and a representative surfactant-containing buffer in microfluidic channels with phaseguide structures oriented at an angle of $90^{\circ}$ to the channel length as function of their height, $h$. The all-polymer chips were fabricated by injection moulding and sealed by ultrasonic welding. Channels with a height of $200 \mu \mathrm{m}$ and widths of $1 \mathrm{~mm}$ or $3 \mathrm{~mm}$ were investigated for five values of $h$ between $8 \mu \mathrm{m}$ and $82 \mu \mathrm{m}$. Phaseguide structures without branches and with branches at angles $\alpha=45^{\circ}, 60^{\circ}$ and $75^{\circ}$ were studied. All phaseguide structures were found able to pin both liquids and the burst pressure was found to increase approximately linearly with the height of the phaseguide from about $100 \mathrm{~Pa}$ to $350 \mathrm{~Pa}$ for water and from about $25 \mathrm{~Pa}$ to $200 \mathrm{~Pa}$ for the buffer. The burst pressure was found not to depend on the channel width and it was only weakly influenced by the presence of a branch on the phaseguide. For phaseguides with a branch, the liquid was always found to burst at the branch location. The burst pressures were compared to those estimated using a simple theory. The knowledge obtained in this study enables simple tuning of liquid spreading and overflow in microfluidic channels by use of phaseguide structures with different heights and it also provides a set of systematic experimental data to be compared with simulations/theory.
\end{abstract}

Keywords: all-polymer chip, phaseguide, injection moulding, ultrasonic welding, hydrostatic burst pressure, topas

PACS: 81.20.Hy, 81.20.Vj, 47.15.G-, 47.15.Rq, 47.80.Fg 


\section{Introduction}

Portable microfluidic devices have attracted significant interest for point-of-care (POC) testing during the last two decades due to their potential of producing fast results at reduced cost. Traditionally, such devices were developed using rapid prototyping techniques, such as casting of polydimethylsiloxane (PDMS) and/or cleanroom micromachining; however, neither technique is well suited for low-cost mass-production (Becker, 2010). Within the last few years, focus has changed to develop devices using techniques and materials that can be directly transferred to an industrial mass-production setting (Mukhopadhyay, 2009).

Recently, we demonstrated rapid shim prototyping and injection moulding of polymer lab-on-a-chip systems that were sealed using ultrasonic welding (Kistrup et al., 2015; Poulsen et al., 2016). In these, the inverse microchannel structures were defined in the shims using micromilling and energy director structures for ultrasonic welding were defined using laser machining. Both injection moulding and ultrasonic welding are widely used techniques for low-cost industrial mass-production and joining of polymer components. In our setting, the injection moulding of a single chip took about 1 min and ultrasonic welding could be completed (including mounting) in about $30 \mathrm{~s}$ per chip. The combination of a shim made by rapid prototyping with injection moulding and ultrasonic welding enabled us to go from design to a 'bag of sealed chips' in as little as 1-2 days (Kistrup et al., 2015).

The advantage but also the curse of lab-on-a-chip systems is the high surface-to-volume ratio that enables fast reactions but also has as a consequence that capillary forces dominate over inertial forces. A well-known issue in lab-on-a-chip systems is the occurrence and trapping of air bubbles at undesired locations. Another challenge in lab-on-a-chip systems has been the integration of valves and pumps to enable multi-chamber microfluidic operations. The advent of phaseguide structures by Vulto et al. presented an attractive approach to the controlled wetting and filling of microfluidic structures to solve these challenges (Vulto et al., 2006). The phaseguide structures are ridges extending from the channel bottom or top which pin the liquid meniscus until the pressure exceeds the burst pressure 
defined by the geometry of the structure and the wetting properties of the liquid. The burst pressure could be tuned by varying the angle between the phaseguide ridge and the sidewall of the channel or by introducing kinks or branches in the phaseguide structure (Trietsch et al., 2011; Yildirim et al., 2014). By use of phaseguide structures, passive liquid valving and the controlled and bubble-free liquid filling of microfluidic chambers of arbitrary shape was demonstrated (Vulto et al., 2011). Trietsch et al. showed controlled filling and emptying of a non-hydrophilic monolithic chip with dead angle sections by use of supporting phaseguides (Trietsch et al., 2011). Vulto demonstrated the spatially and sequentially controlled phaseguide overflow using a passive valve approach (Vulto et al., 2011). Yildirim presented liquid routing using a hierarchy of phaseguides with different burst pressures (Yildirim et al., 2014). Moreover, Phurimsak et al. demonstrated the use of phaseguides to layer different liquids in a single chamber to facilitate an enzyme-linked immunosorbent assay (Phurimsak et al., 2014). Finally, Gottheil et al. demonstrated the use of phaseguide structures and capillary valves in an injection moulded polymer chip to form a four-chamber microfluidic device for a sandwich immunoassay (Gottheil et al., 2014).

The chips in all of the above studies except for (Gottheil et al., 2014) were fabricated using photolithography, which leads to highly well-defined phaseguides of the same height to keep the fabrication simple. When structures are defined by milling and fabricated by injection moulding, it is easy to modify the height of the structures while maintaining a constant geometry; however this has not yet been studied systematically in the literature. Here, we present a systematic experimental analysis of the burst pressure of phaseguide structures in polymer chips, fabricated by injection moulding using a micromilled shim, as function of their height, $h$, and we study the dependence on the channel width and the effect of including branches to the phaseguides. 


\section{Theory}

Burst pressure measurements are used to determine when the liquid will overflow the phaseguides and propagate through the chip. These pressures can be estimated analytically for simple geometries. Below, we briefly consider the dominant forces in microfluidic systems. The Young-Laplace equation, Eq. (1), expresses the pressure difference over an interface between two phases (here liquid-gas) with surface tension $\gamma$ characterized by radii of curvature $R_{1}$ and $R_{2}$ as

$$
\Delta p=\gamma\left(\frac{1}{R_{1}}+\frac{1}{R_{2}}\right)
$$

Note, that with this definition, the pressure is higher in the medium in which the centers of the radii of curvature are placed (the convex side of the interface). For a channel with a rectangular cross-section of width $W$ and height $H$ and which is formed by a structured moulded part sealed by a flat foil, Eq. (1) can be written in terms of the contact angles $\theta_{\mathrm{m}}$ and $\theta_{\mathrm{f}}$ of the liquid to the moulded part (m) and the foil (f), respectively, as

$$
\Delta p_{\text {cap }}(H)=\gamma\left(\frac{2 \cos \theta_{\mathrm{m}}}{W}+\frac{\cos \theta_{\mathrm{m}}+\cos \theta_{\mathrm{f}}}{H}\right) \approx \gamma \frac{\cos \theta_{\mathrm{m}}+\cos \theta_{\mathrm{f}}}{H},
$$

where the latter expression is valid for $W \gg H$. Note, that $\Delta p_{\text {cap }}$ in Eq. (2) denotes the increase in pressure upon passing through the liquid-gas interface, i.e., $\Delta p_{\text {cap }}>0$ drives capillary filling of the channel whereas $\Delta p_{\text {cap }}<0$ does not. Consequently, depending on whether $\cos \theta_{\mathrm{m}}+\cos \theta_{\mathrm{f}}>0$ (wetting) or $<0$ (non-wetting), the capillary pressure in Eq. (2) may assist or hinder the filling of the microfluidic channel. The phaseguide of height $h$ first defines a contraction of the channel and subsequently an expansion (figure 1). Above a wide phaseguide, a non-wetting liquid will experience a higher capillary pressure, $\Delta p_{\text {cap }}(H-h)$, which may prevent the liquid from reaching the back edge of the phaseguide. When the liquid-gas interface has passed the phaseguide, the liquid will again experience the capillary pressure $\Delta p_{\text {cap }}(H)$. In the experimental studies below, the interface was observed to pin at the back edge of the phaseguide in all experiments. 


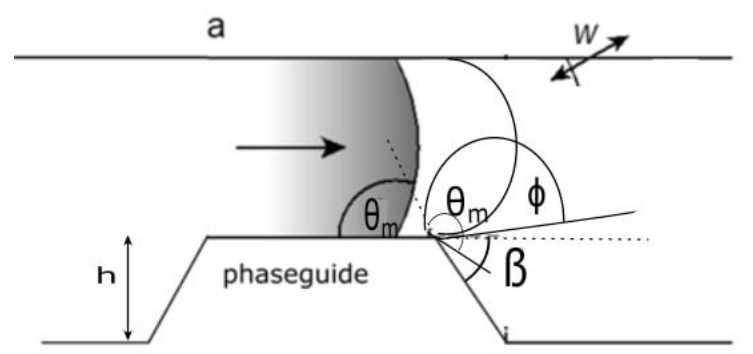

Figure 1: Side-view sketch of channel cross-section with a liquid passing over a phaseguide (left to right), where pinning is achieved through a sudden expansion of the geometry in the moulded part (top in figure). The parameters $\theta_{\mathrm{m}}, \alpha$, and $\phi$ describe the liquid contact angle to the moulded part, the expansion angle, and the effective contact angle, respectively. $W$ and $h$ denote the width of the channel and height of the phaseguide.

When the liquid-gas interface has proceeded to the back edge of the phaseguide, it may be pinned. At this edge, the phaseguide defines an abrupt expansion of the channel with an angle $\beta$ to horizontal (side-view in figure 1). Following the arguments of (Cho et al., 2007) the interface contact angle to the expanded region, $\phi$, at the expansion is smaller than the advancing contact angle, $\theta_{\mathrm{m}}$, of the expanded region and the interface is pinned if $\phi \leq \theta_{\mathrm{m}}+\beta$. For the structure in figure 1, the pressure that we need to apply to overcome the pinning at the edge of the expansion is

$$
p_{\text {pin }}=-\gamma\left(\frac{2 \cos \theta_{\mathrm{m}}}{W}+\frac{\cos \left(\min \left\{\theta_{\mathrm{m}}+\beta, 180^{\circ}\right\}\right)+\cos \theta_{\mathrm{f}}}{H-h}\right)
$$

Eq. (3) determines the calculated burst pressure of the structure. A value of $\boldsymbol{p}_{\text {pin }}>\mathbf{0}$ indicates that the liquid-gas interface is pinned at the edge (pinning condition), whereas $\boldsymbol{p}_{\text {pin }}<\mathbf{0}$ indicates that the liquid-gas interface will pass the edge without being pinned. In the devices investigated in the present work, the nominal expansion angle was $\boldsymbol{\beta}=\mathbf{7 5}^{\circ}$. For $\boldsymbol{W} \gg \boldsymbol{H}-\boldsymbol{h}$, it can be observed by inspection of Eq. (3) for $\boldsymbol{\theta}_{\mathbf{m}}<\mathbf{1 0 5}^{\circ}$ that a pinning requirement is that $\cos \left(\boldsymbol{\theta}_{\mathbf{m}}+\mathbf{7 5}^{\circ}\right)+\cos \boldsymbol{\theta}_{\mathbf{f}}<\mathbf{0}$. If this is no longer fulfilled, the sidewall contribution will reduce $\boldsymbol{p}_{\text {pin }}$ for $\boldsymbol{\theta}_{\mathbf{m}}<\mathbf{9 0}$ and increase $\boldsymbol{p}_{\text {pin }}$ for $\boldsymbol{\theta}_{\mathbf{m}}>\mathbf{9 0}^{\circ}$. The full Eq. (3) was used in all calculations below. 


\section{Methods}

\subsection{Chip fabrication and design}

The all-polymer chip consisted of two parts: a main part in cyclic olefin-copolymer (COC) polymer (TOPAS grade 5013L-10), fabricated by injection moulding and a $0.254 \mathrm{~mm}$ COC foil (TOPAS grade 5013S-04) both polymers from TOPAS Advanced Polymers GmbH, Frankfurt-Höchst, Germany. Injection moulding was conducted on an Engel Victory 80/45 Tech injection moulder (ENGEL, Schwertberg, Austria), featuring the negative counter-part of the channel layout on one side of the injection moulding tool and a luer-layout counter-part with through-holes on the other (Andresen et al., 2010). The machine was set to run an isothermal program with injection and mould temperatures of $270^{\circ} \mathrm{C}$ and $120^{\circ} \mathrm{C}$, respectively. During injection moulding, a pressure of 1766 bar was applied for 5 s and finally the chip was cooled for 30s.

The mould insert/negative counterpart, designed with Autocad Inventor 2016, was micro-milled with a three-axis Mini-Mill/3 mill frame fitted with a Nakanishi E3000C spindle and controller (MinitechMachinery, Norcross, Georgia) on a $2 \mathrm{~mm}$ thick sheet of aluminum alloy 2017. Phaseguides were fabricated in a final milling step using an engraving tool (DIXI polytool 7009, art 976370, 30, $\mathrm{D} 1=50 \mu \mathrm{m})$. This tool is conical with an angle of each of its sides to vertical of $15^{\circ}$ and it has a flat tip with a diameter of $50 \mu \mathrm{m}$. The last step before injection moulding was the creation of energy directors, special protrusions that allow localizing the vibrations of the sonotrode of the ultrasonic welder to the areas were welding is required, via the use of a microSTRUCT vario picosecond laser (3D-Micromac AG, Chemnitz, Germany) as described by (Poulsen et al., 2016). To complete the chip, the injection moulded top part was bonded to the bottom COC sheet using a Telsonic USP4700 ultrasonic welder (Telsonic, Erlangen, Germany) as described previously ( Poulsen et al., 2016).

The main part featured the chip layout with seven channels in parallel, each with its own luer-fitted inlet. Each channel had a height of $H=200 \mu \mathrm{m}$ and contained a sequence of five phaseguides of increasing nominal heights $h_{\text {nom }}==20 \mu \mathrm{m}, 40 \mu \mathrm{m}, 60 \mu \mathrm{m}, 80 \mu \mathrm{m}$ and $100 \mu \mathrm{m}$. All phaseguides 
were straight and orthogonal to the channel length $\left(90^{\circ}\right)$. One channel had a width of $W=1 \mathrm{~mm}$ and did not feature any branches on the phaseguides. The remaining six channels consisted of three channels with $W=1 \mathrm{~mm}$ and three channels with $W=3 \mathrm{~mm}$. The phaseguides in each of these channels had a centrally placed branch forming an angle of $\alpha=45^{\circ}, 60^{\circ}$ or $75^{\circ}$ (see figure 2a). It was decided not to study a $90^{\circ}$ branch, due to the similarity to the 'no branch' one with $90^{\circ}$ angle to the sidewalls. The overall chip structure, including the luer inlets, is illustrated in figure $\mathbf{2 b}$.

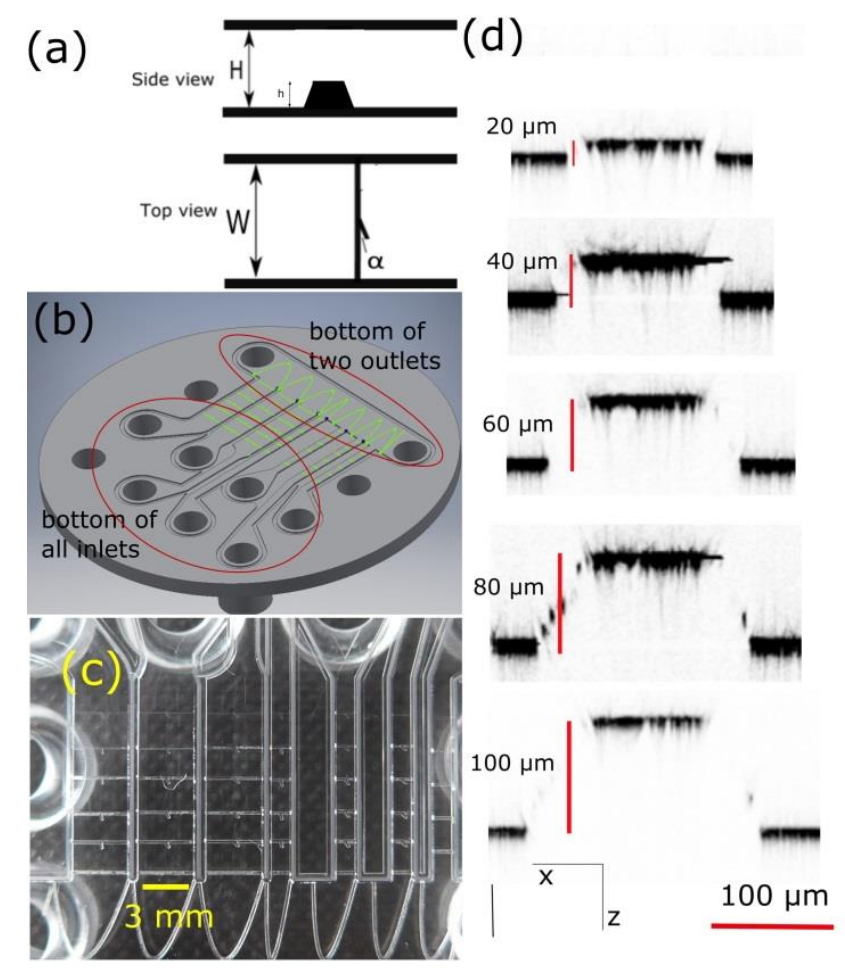

Figure 2: (a) Side and top views of a channel (height $H$, width $W$ ) with $90^{\circ}$ phaseguide structure (height $h$ ) including a branch placed centrally at an angle $\alpha$. (b) Layout of seven-channel injection moulded chip with integrated luer connectors and a common waste chamber. (c) Photograph of the fabricated chip prior to ultrasonic welding showing the parallel layout of the channels containing the phaseguides with branches(first six channels from left) and one without branches (last channel on the right), (d) Cross-sections of all phaseguide profiles indicating the measured heights as obtained by confocal microscopy.

\subsection{Test liquids}

Two different liquids were used in experiments: (1) water obtained from a Millipore MilliQ lab water system, and (2) a mixture of phosphate buffer saline containing $0.01 \%(\mathrm{~V} / \mathrm{V})$ of surfactant Tween 20 
and $0.1 \%(\mathrm{~V} / \mathrm{V})$ of protein bovine serum albumin (referred to as PBST-BSA). The latter liquid was used as a representative buffer for analysis of biological samples. To facilitate visualization of the liquid in the channels, Brilliant Blue R dye was added to both solutions. The PBST-BSA reagents and the dye were purchased from Sigma-Aldrich. Densities of the dyed solutions were measured at room temperature to $\rho_{\text {water }}=998 \mathrm{~kg} / \mathrm{m}^{3}, \rho_{\text {PBST-BSA }}=1005 \mathrm{~kg} / \mathrm{m}^{3}$.

\subsection{Contact angle and interfacial tension measurements}

All measurements were performed on a Krüss DSA10 Contact Angle Measuring System (Krüss GmbH, Hamburg, Germany) with the associated DSA1 v1.9 software. Measurements, including both advancing and static contact angles, were performed using the "sessile drop" method and fitting the obtained images using the "tangent 2" method in the instrument software. Liquid-air interfacial tension measurements were performed using the "pendant drop" method.

\subsection{Burst pressure measurements}

Hydrostatic burst pressures were determined by raising one end of a peroxide cured silicone tubing $(\mathrm{OD}=10 \mathrm{~mm}, \mathrm{ID}=6 \mathrm{~mm})$, filled with the test liquid, connected through the other end to the chip to a height $\Delta z$ above chip level using a Thorlabs LTS150 motorized stage (Thorlabs, Newton, NJ, USA) (schematic in figure 3a and picture in figure S1, ESI). In the experiments, the chips were mounted with the luer connectors facing downwards. An experiment was performed by first adjusting $\Delta z$ to zero. Then, the tubing was raised at $1 \mathrm{~mm} / \mathrm{s}$ while carefully monitoring the position of the liquid meniscus in the channel. When the meniscus advanced in the channel, the stage was paused and the meniscus was allowed to pin at the next phaseguide (figure $\mathbf{3 b}$ ) after which the stage motion continued. Measurements were performed on three different chips, which were rinsed and dried before conducting another set of measurements. The applied burst pressure was determined as

$$
p_{\text {burst }}=\rho g \Delta z
$$


where $\rho$ is the density of the liquid, $g=9.82 \mathrm{~m} / \mathrm{s}^{2}$ is the gravitational acceleration.

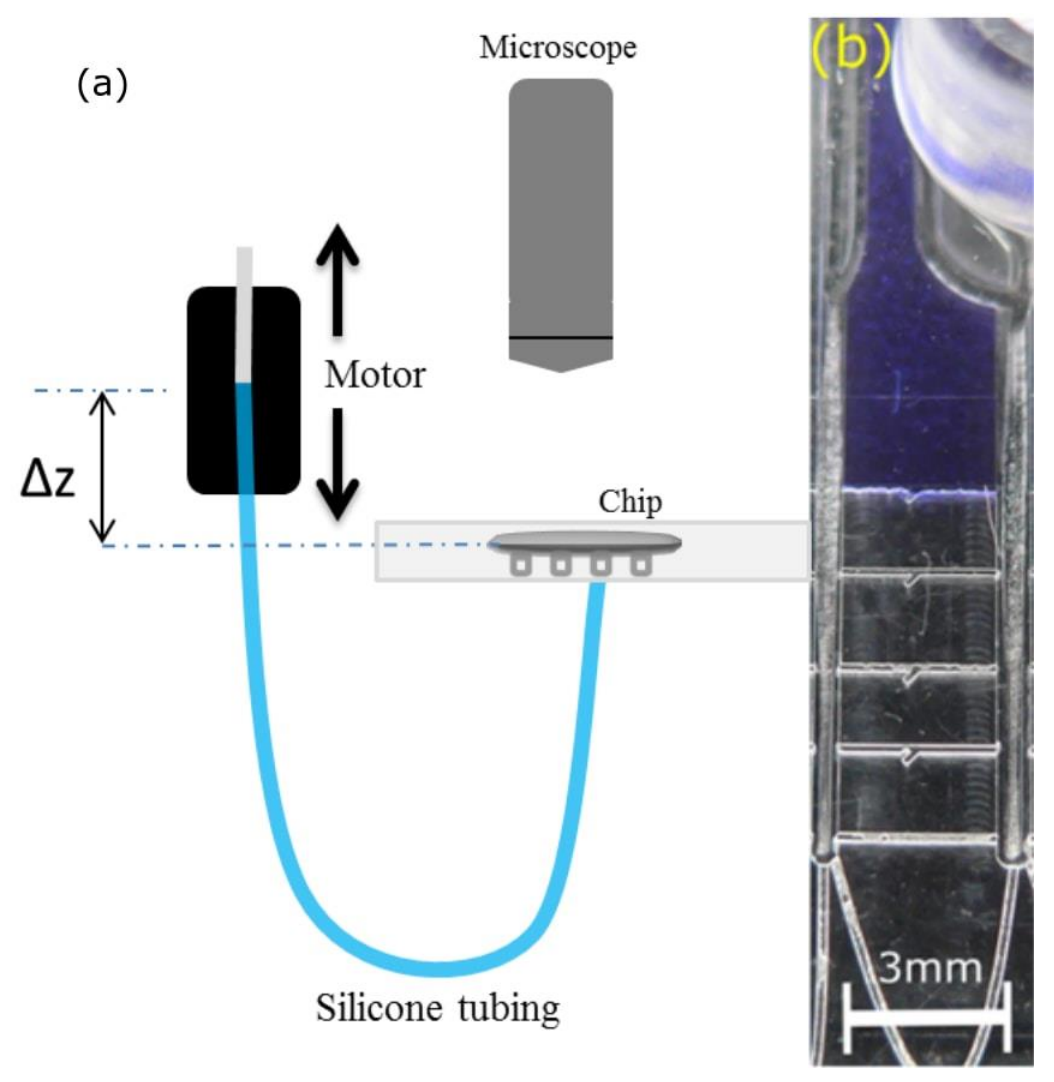

Figure 3: (a) Schematic of the setup, with the chip (and luer connectors) facing downwards and attached to the tubing. The other end of the liquid filled tube was mounted in a linear motor stage such that its height could be changed at constant velocity. In an experiment, the tube was first raised to fill the chip until it pinned at the first phaseguide. Then, the position of the stage was adjusted to make the height of the liquid-air interface in the open end of the tube coincide with the top of the injection moulded part (top of the channel) defining $\Delta z=0$. Subsequently, the motor stage was set in motion at constant velocity and the time at which the liquid meniscus burst and reached the next phaseguide was recorded. This continued for all phaseguides in a channel. (b) Picture of the liquid meniscus pinned at the second phaseguide $\left(h_{\text {nom }}=40 \mu \mathrm{m}\right)$ in a $3 \mathrm{~mm}$ wide channel.

\subsection{Microscopy}

The geometries of the fabricated chips and phaseguides were investigated using a Zeiss LSM 700 confocal microscope $(20 \times / 0.5, z$-stack images, and $1.590 \mu \mathrm{m}$ sections $)$. Images were analysed using ImageJ v. 1.47 and geometrical parameters of the relevant chip cross-sections in the $z$-direction were obtained from cross-sections of the stacked images.

The quality of the bonding of the channel lid was investigated using a standard stereo microscope (MZ

FLIII, Leica Microsystems GmbH, Wetzler, Germany) equipped with a CCD camera (DFW-X710, 
Sony, Tokyo, Japan). In the experiments, the chips were filled with PBST-BSA with added Brilliant Blue $\mathrm{R}$ dye. The quality of the bonding was investigated by visual inspection of the colour transition from the channel to the area just outside the channel.

\section{RESULTS}

\subsection{Physical characterization}

The advancing contact angles were found to differ for the foil $\left(\theta_{\mathrm{f}}\right)$ and the injection moulded part $\left(\theta_{\mathrm{m}}\right)$ in which the channels were defined. For the dyed water, we measured $\theta_{\mathrm{m}}=96 \pm 2^{\circ}$ and $\theta_{\mathrm{f}}=74 \pm 4^{\circ}$ and for the PBST-BSA solution we measured $\theta_{\mathrm{m}}=83 \pm 2^{\circ}$ and $\theta_{\mathrm{f}}=76 \pm 6^{\circ}$, where the stated uncertainties indicate the standard deviation on the mean $(n=10)$. The results for the foil showed a larger variability due to inhomogeneity of the surface and were generally lower than those for the moulded part. We speculate that this could be due to residues on the foil from the fabrication. The liquid-air surface tension $\gamma$ was measured to $73 \mathrm{mN} / \mathrm{m}$ for the dyed water and $35 \mathrm{mN} / \mathrm{m}$ for the PBST-BSA, respectively. These values are consistent with those reported in the literature for similar solutions (Niño and Patino, 1998). The contact angle for PBST-BSA was further investigated, due to its modification of the surface wettability, once a chip had been exposed to BSA. This hypothesis was verified by contact angle measurements on substrates given an overnight exposure to PBST-BSA, where we found a reduction of the contact angle from $83^{\circ}$ to around $65^{\circ}$.

Figure 2c shows an optical image (top view) of the channels with phaseguide taken prior to ultrasonic welding of the channel lid. All channel and phaseguide geometries were investigated by confocal microscopy to verify the channel and phaseguide dimensions. It was found that the channel width $W$ and height $H$ corresponded to their nominal values and also that the geometries of the phaseguide branches matched those on the design. The angle of the phaseguide sidewall to horizontal was measured to $\beta=75^{\circ}$ as expected from the opening angle of the engraving tool ( $15^{\circ}$ to vertical). Figure 2d shows cross-sections of the phaseguide ridges as determined by confocal microscopy. A 3D view of a phaseguide is given in Section S2, ESI. The heights of the phaseguide ridges were also 
investigated by scanning probe profilometry. Analysis of the profiles (Section S3, ESI) showed that the measured values of $h$ were systematically $12 \mu \mathrm{m}$ lower than their nominal values $h_{\text {nom }}$, such that the actual five values of $h$ in the sequence of phaseguides were $h=8 \mu \mathrm{m}, 28 \mu \mathrm{m}, 48 \mu \mathrm{m}, 68 \mu \mathrm{m}$ and $88 \mu \mathrm{m}$. This deviation is attributed to an offset in the $z$-zeroing of the engraving tool during the micromilling fabrication of the shim. The measured values will be used in the plots and the analysis below. The investigation also indicated that one of the structures was not realized as expected due to left-over particles in the smallest phaseguide cavities in the master insert used for the injection moulding (Section S1, ESI). The results for this structure were discarded in the analysis.

Standard microscopy of chips filled with dyed PBST-BSA was used to study the quality of the channel sealing. Due to fabrication tolerances, the approximately $10 \mu \mathrm{m}$ high energy directors were defined as lines surrounding the channels with a lateral spacing to the channel of $205 \mu \mathrm{m}$. In the images (Section S4, ESI), we observed a sharp transition in colour between the channel and the region outside the channel with no detectable colouring in the narrow region between the channel and the welded energy directors. These observations are consistent with those of Poulsen et al. by confocal fluorescence imaging of a fluorescent dye filled into channels using the same energy directors and ultrasonic welding procedure. This indicates that the ultrasonic welding provided a leak-tight sealing of the channels and also that the gap between the moulded part and the foil after welding was negligible.

\subsection{Burst pressure measurements}

Insertion of the measured contact angles and surface tension in Eq. (2) reveals capillary pressures of $\Delta p_{\text {cap }}(H)$ of $62 \pm 2 \mathrm{~Pa}$ for water and $63 \pm 2 \mathrm{~Pa}$ for PBST_BSA and thus the channels are expected to fill spontaneously with the liquid. This was in agreement with the experimental observations. Further, we find by insertion of the dimensions and contact angles in the expression for $p_{\text {pin }}$ in Eq. (3) that both liquids fulfill the requirement for pinning of the liquid meniscus. Again, this was found to agree with 
experimental observations and it is therefore meaningful to determine the burst pressures for the phaseguides.

The burst pressure $p_{\text {burst }}$ at which the pinned liquid meniscus moved to the next phaseguide structure was determined experimentally as function of the relative height $h / H$ of phaseguides with the indicated angles $\alpha$ of the branch for channels with $W=1 \mathrm{~mm}$ and $W=3 \mathrm{~mm}$ for water and PBSTBSA, respectively.
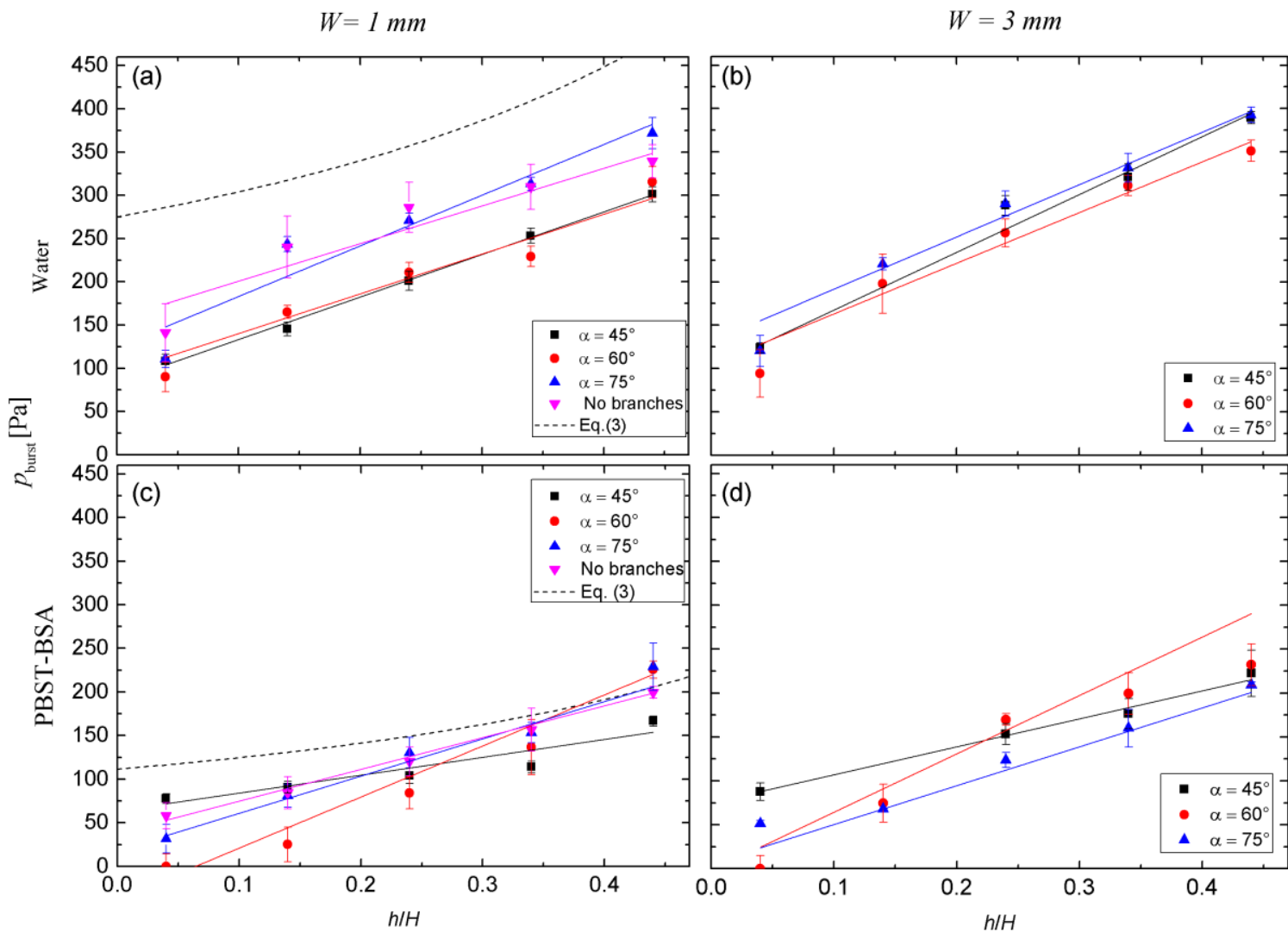

Figure 4: Burst pressure $p_{\text {burst }}$ measured as function of $h / H$ for phaseguides with branches at the indicated angles and the indicated channel widths. Results for $W=1 \mu \mathrm{m}$ also include a channel without a branch on the phaseguide. Panels (a) and (b) show results obtained for water and panels (c) and (d) show results obtained for the PBST-BSA solution. Error bars in all graphs refer to the standard deviation of the mean ( $n=3$ for PBST-BSA and $n=9$ for water). Solid lines are linear fits of each dataset listed in the text included to guide the eye. The dashed lines are plots of Eq. (3) using the measured values of contact angles and liquid-air surface tension given in the text. 
Figure 4a shows measured values of $p_{\text {burst }}$ for water in a channel with $W=1 \mathrm{~mm}$ as function of $h / H$ for a phaseguide without a branch and for phaseguides with branches with the indicated values of $\alpha$. Note, that a pressure of $100 \mathrm{~Pa}$ corresponds to a liquid column with a height of about $10 \mathrm{~mm}$. Error bars were determined as the standard deviation on the mean from three repeated experiments on three separate chips $(n=9)$. The values of $p_{\text {burst }}$ were observed to increase systematically with $h / H$ in an approximately linear fashion from about $100 \mathrm{~Pa}\left(\approx 10 \mathrm{~mm} \mathrm{H}_{2} \mathrm{O}\right)$ for $h=8 \mu \mathrm{m}$ to about $350 \mathrm{~Pa}$ for $h=88$ $\mu \mathrm{m}$. Below, we describe them in terms of a simple empirical linear model and compare later to the expectations based on the theory presented in Section 2. The results could be divided into two groups corresponding to phaseguides with low-angle branches $\left(\alpha=45^{\circ}\right.$ and $\left.60^{\circ}\right)$ or phaseguides with no branch or a high-angle branch $\left(\alpha=75^{\circ}\right)$. The results within each of these groups were found to be identical (one-way ANOVA $\mathrm{F}(1,132)=3.51, \mathrm{p}=.06$ for the first grouping and one-way ANOVA $\mathrm{F}(1,132)=2.87, \mathrm{p}=.07$ for the second grouping) and the results for the low-angle branches were found to be systematically lower than those for the other group. The solid lines in figure. 4a are linear fits for the two groups (to guide the eye) to

$$
p_{\text {burst }}=p_{0}+p_{\text {slope }} \cdot(h / H) \text {. }
$$

The parameters obtained from fits of Eq. (5) to the separate series of data for the different phaseguide branch types are given in table 1. It should be noted that the experiment to experiment variation (and hence the error bars) was larger for the phaseguide with no branches than for those with branches. Moreover, in all experiments on phaseguides with branches, the liquid interface was observed to always overflow a phaseguide at the branch location. 
Table 1: Values of $p_{0}$ and $p_{\text {slope }}$ obtained from fits of $p_{\text {burst }}=p_{0}+p_{\text {slope }} \cdot(h / H)$ to the values measured for the conditions indicated below. The stated uncertainties indicate the standard deviations obtained from the fits.

\begin{tabular}{cc|cc|cc}
\hline \multicolumn{2}{c|}{ Geometry } & \multicolumn{2}{|c|}{ Water } & \multicolumn{2}{c}{ PBST-BSA } \\
$W[\mathrm{~mm}]$ & Phaseguide branch & $p_{0}[\mathrm{~Pa}]$ & $p_{\text {slope }}[\mathrm{Pa}]$ & $p_{0}[\mathrm{~Pa}]$ & $p_{\text {slope }}[\mathrm{Pa}]$ \\
\hline 1 & $\alpha=45^{\circ}$ & $84 \pm 5$ & $492 \pm 17$ & $63 \pm 12$ & $205 \pm 42$ \\
1 & $\alpha=60^{\circ}$ & $94 \pm 17$ & $460 \pm 71$ & $-38 \pm 15$ & $586 \pm 46$ \\
1 & $\alpha=75^{\circ}$ & $124 \pm 30$ & $586 \pm 120$ & $18 \pm 11$ & $427 \pm 43$ \\
1 & no branch & $158 \pm 25$ & $435 \pm 75$ & $63 \pm 12$ & $205 \pm 42$ \\
\hline 3 & $\alpha=45^{\circ}$ & $100 \pm 11$ & $667 \pm 42$ & $74 \pm 5$ & $318 \pm 22$ \\
3 & $\alpha=60^{\circ}$ & $104 \pm 23$ & $584 \pm 66$ & $-2 \pm 31$ & $665 \pm 129$ \\
3 & $\alpha=75^{\circ}$ & $131 \pm 13$ & $603 \pm 46$ & $6 \pm 7$ & $443 \pm 53$ \\
\hline
\end{tabular}

Figure $4 \mathbf{b}$ depicts the corresponding results for water in a channel with $W=3 \mathrm{~mm}$. For this channel width, a phaseguide without a branch was not fabricated. The burst pressures showed values and a behaviour similar to that for $W=1 \mathrm{~mm}$, but the values were found to be slightly larger for the lowangle branches $\left(\alpha=45^{\circ}\right.$ and $\left.60^{\circ}\right)$, whereas the burst pressures for the high-angle branch $\left(\alpha=75^{\circ}\right)$ were very similar to those obtained for $W=1 \mathrm{~mm}$. The solid lines are linear fits of Eq. (5) to the data to guide the eye. The chip geometry and measured burst pressures were found to be reproducible and no systematic variation between burst pressures measured repeatedly on the same chip $(n=3)$ and on different chips $(n=3)$ was observed (see Section S5, ESI). Thus, the observed variation between repeated experiments is likely due to the experimental reproducibility of the burst pressure measurements.

Figures 4c-d show the corresponding results for PBST-BSA for $W=1 \mathrm{~mm}$ and $W=3 \mathrm{~mm}$, respectively. In both cases, the behaviour was similar to that obtained for water, but the burst pressures were lower and a possible grouping of the results was less obvious. The burst pressures were found to either slightly depend or not depend at all on the channel width. For both channel widths, the slope for $\alpha=45^{\circ}$ was observed to be lower than for phaseguides with $\alpha>45^{\circ}$. The lines are linear fits to groups of data consisting of $\alpha=45^{\circ}$ and the other phaseguides, respectively (to guide the eye). It should be noted that the PBST-BSA measurements were performed on three different chips $(n=3)$, and 
that the behaviour changed irreversibly once a channel had been primed with the PBST-BSA solution. For this reason, repeated measurements on the same chip were not performed for PBST-BSA.

\section{Discussion}

\subsection{Reproducibility of burst pressures and effect of phaseguide branch}

The studies of the fabricated chip geometries revealed that the channel and phaseguide geometries could be fabricated consistently with dimensions identical to their nominal values, except for the phaseguide height, where values were systematically $12 \mu \mathrm{m}$ lower than their nominal values due to an offset in the zeroing of the engraving tool. Moreover, we found that the chips could be sealed without leaks using ultrasonic welding. The measured burst pressures were generally found to be reproducible and for water no systematic variation was observed between repeated experiments, on the same chip, and between nominally identical chips (see Section S5, ESI). Therefore, the presented method allowed for the fabrication of a large number of single-use chips within a few days with burst pressures that were reproducible within the experimental uncertainty.

The branches on the phaseguide were found to have only a modest impact on the measured burst pressures, which was most pronounced for water in channels with $W=1 \mathrm{~mm}$. Their main function was to define a specific point of overflow to provide a reproducible and well-defined continued filling of the channel. A related observation from figure 4 is that phaseguides with branches with $\alpha=45^{\circ}$ (in particular) and $\alpha=60^{\circ}$ generally produced results with lower variation compared to phaseguides with no branches or branches with $\alpha=75^{\circ}$. In our work, this indicates that phaseguides with branches with $\alpha=45^{\circ}$ provided the best control and reproducibility of the liquid overflow.

The measurements using PBST-BSA were also found to be reproducible from chip-to-chip on a level comparable to those obtained with water. However, repeated measurements on the same chip were not reproducible, due to the decrease in contact angle as explained in the results part. 


\subsection{Comparison to analytical modelling}

The analytical model presented in Section 2 only concerns the phaseguide structures without branches and we therefore restrict the comparison to the results obtained for channels $W=1 \mathrm{~mm}$. It is noted, however, that as $W \gg H$ for both channel widths, the theory predicts a minimal dependence on the channel width in agreement with the experimental observations. The dashed lines in figures $\mathbf{4 a}$ and $\mathbf{4 c}$ show the pinning pressure $p_{\text {pin }}$ vs. $h / H$ calculated by insertion of the measured values of the surface tension and contact angles in Eq. (3) for the two liquids. In both cases, the calculated pinning pressure is found to be comparable to (but somewhat overestimating) the measured burst pressures and it shows an increase with $h / H$ comparable to that observed experimentally. Moreover, the change in pinning pressure with the change of liquid is comparable to (but slightly underestimating) that observed experimentally. However, it is clear that the pinning pressure calculated from the measured parameters does not provide a quantitative description of the measured values. It is noted that it was found to be an ill-defined problem to fit Eq. (3) to the available measured data and this option was therefore not further pursued.

Generally, we observed that the theory tended to overestimate $p_{\text {burst }}$ at low values of $h / H$ and thus underestimated the slope of $p_{\text {burst }}$ vs. $h / H$. Several factors could contribute to the observed deviations:

First, we note that the roughness of the channel introduced during the milling process of the shim may cause a variability in the effective contact angle. As both liquids were observed to wet the substrate (Wenzel wetting state), a roughness will enhance the already existing wetting properties and thus make a wetting liquid more wetting (reduction of contact angle) and a non-wetting liquid less wetting (increase of contact angle). The contact angle measurements on the injection moulded part, however, were performed on an area where the shim had been subject to milling and thus we expect that the measured values are representative for those of the fluid channels. 
Second, we note a lowering of the contact angle and surface tension could in principle contribute to the observed deviation. However, great care was taken in the experiments to use different tubing for the water and PBST-BSA such that the values for the contact angle and surface tension measured for water were valid also for the on-chip experiments.

Third, the actual profile of the phaseguide could deviate from the ideal profile assumed in the calculations, for example by the edge being more rounded or by having a variation in the height created by milling burrs left in the cavity in the shim, which is the inverse of the phaseguide structure (Section S2, ESI). This could weaken the pinning ability of the phaseguide and thus reduce the measured burst pressure compared to the calculated one. This effect is random in nature but reproducible for the same phaseguide from chip to chip as they are made from the same shim. The systematic variation of the results with phaseguide geometry indicates that the effect is small as we would otherwise anticipate a substantial random variation of the results with the phaseguide geometry.

Finally, the theory considers a liquid meniscus that can bulge freely near the pinning point of the phaseguide when the liquid is non-wetting. This requires that the height of the phaseguide is sufficient to avoid contact between the liquid-air interface and the channel bottom after the phaseguide. When the height of the phaseguide is low, however, this may not be fulfilled in practice and this may cause a reduction of the measured burst pressure compared to the calculated one.

At this point, we therefore simply note that the simple theory in Eq. (3) is able to predict the magnitude of the burst pressure and the trends of the burst pressure with changing liquid/wetting properties and the channel geometry, but also that for the determined experimental parameters the agreement between Eq. (3) and the experimentally determined burst pressures is not quantitative.

\subsection{Comparison to literature}

We generally observed that the burst pressures for the investigated straight $90^{\circ}$ phaseguides without a branch were close to those with a branch for $\alpha=75^{\circ}$ and higher than those for phaseguides with 
lower angle branches $\left(\alpha=45^{\circ}\right.$ and $\left.60^{\circ}\right)$. Moreover, the branches were found to define the point at which the liquid meniscus burst through the phaseguide. These results are qualitatively consistent with those obtained by (Vulto et al., 2011) on lithographically defined phaseguides with a width and height of about $30 \mu \mathrm{m}$ and $\beta \approx 90^{\circ}$, but the effect of the branch on the phaseguide is less pronounced in our study. A possible cause of this can be the different fabrication methods and materials where the cleanroom fabrication provides structures with a sharper and a better controlled geometry.

Yildirim et al. presented an analytical model for the pinning behaviour of a liquid in a channel with a highly hydrophilic top on phaseguides with a square cross-section with focus on the effect of the angle of the phaseguide to the channel sidewall (Yildirim et al., 2014). Based on their more elaborate model, they concluded that the pinning behaviour was dominated by the horizontal geometry of the phaseguide. They found good agreement between their model and an experimental study in which the phaseguide angle to the channel sidewall was varied from $50^{\circ}$ to $90^{\circ}$, but only a single channel width was investigated. The present study differs from their study in the respect that we have used a fixed angle of the phaseguide to the sidewall of $90^{\circ}$, our phaseguides have a non-square cross-section and the materials used by us have similar wetting properties in the entire channel. Moreover, we investigated the phaseguide burst pressure as function of the phaseguide height rather than its angle to the sidewall. In our studies, we found that the width of the channel had minimal influence on the result. Further studies are needed to shed more light onto whether these observations are in agreement or in conflict.

Considering previous literature, there is a need for further systematic theoretical studies of the burst pressure of phaseguides in order to reconsider limitations in the present models and develop new models which include the influence of a wider variety of parameters to cover different fabrication techniques. These parameters include the wetting properties of the liquid (wetting/non-wetting), the properties of the materials and the complete geometry of the phaseguide as well as the impact of branches and other supporting structures. 


\subsection{Consequences for applications}

Micromilling and injection moulding allow for the creation of phaseguides of different heights keeping all other geometries constant as an alternative or supplement to varying the angle between the phaseguide structure to the channel sidewall. This can be recognized as a step in the creation of an allpolymer chip with different chambers for clinical diagnostics and can be used to tailor a sequence of burst pressures to suit a given application. For example, the phaseguides can be used to provide a controlled sequential filling of chambers with a significantly reduced risk of trapping of air bubbles (Phurimsak et al., 2014; Yildirim et al., 2014).

\section{Conclusion}

We have presented the fabrication of an all-polymer microfluidic chip with integrated straight capillary stop phaseguide structures forming an angle of $90^{\circ}$ to the channel sidewalls. Single-use chips were fabricated by injection moulding of a part with structures defined in a micromilled shim combined with chip sealing by ultrasonic welding. Both techniques are compatible with low-cost mass production. We measured the burst pressures of the phaseguides as function of their height for phaseguides without and with branches at different angles in channels of two different widths for water and for a representative buffer (PBST-BSA). The results were found to be reproducible and showed a burst pressure that increased approximately linearly with the height of the phaseguide. It was determined that the channel width does not affect significantly the burst pressure. A branch on the phaseguide was found to provide a well-defined burst point and phaseguides with a low-angle branch generally had slightly lower burst pressures than those without a branch or with a branch at a high angle. Results were found to be of the same magnitude but somewhat lower than the predictions of a simple analytical model.

The present work addressed a hitherto not studied parameter, the height of the phaseguide, which can easily be varied in chips made by injection moulding from a shim fabricated by milling. Most of the 
past studies of phaseguides in the literature were based on cleanroom fabricated structures where each layer requires a new mask and thus makes it tedious to vary the height of the phaseguide within a microfluidic chip. Moreover, the phaseguides in the present study also differ from the past literature by having a non-square cross-section with slanted sides. Thus, in addition to presenting an alternative approach to controlling the burst pressure by varying the height of the phaseguide in a massproducible microfluidic system, the present work also fills a gap in the experimental data in the literature on phaseguides that may inspire to further theoretical work on the burst pressure of phaseguides. Our own further work will focus on using phaseguides to form multichamber microfluidic systems for health diagnostics.

\section{ACKNOWLEDGMENTS}

This work was supported by DFF projects 4005-00116 and 4184-00121.

\section{REFERENCES}

Andresen, K.O.Ø., Hansen, M., Matschuk, M., Jepsen, S.T.S.T.S.T., Sørensen, H.S., Utko, P., Selmeczi, D.D., Hansen, T.S., Larsen, N.B., Rozlosnik, N., Taboryski, R., Sorensen, H.S., 2010. Injection molded chips with integrated conducting polymer electrodes for electroporation of cells. J. Micromechanics Microengineering 20, 55010. doi:10.1088/0960-1317/20/5/055010

Becker, H., 2010. Mind the gap! Lab Chip 10, 271-273. doi:10.1039/B925993G

Cho, H., Kim, H.Y., Kang, J.Y., Kim, T.S., 2007. How the capillary burst microvalve works. J. Colloid Interface Sci. 306, 379-385. doi:10.1016/j.jcis.2006.10.077

Gottheil, R., Baur, N., Becker, H., Link, G., Maier, D., Schneiderhan-Marra, N., Stelzle, M., 2014. Moving the solid phase: A platform technology for cartridge based sandwich immunoassays. Biomed. Microdevices 16, 163-172. doi:10.1007/s10544-013-9816-2

Kistrup, K., Poulsen, C.E., Hansen, M., Wolff, A., 2015. Ultrasonic welding for fast bonding of selfaligned structures in lab-on-a-chip systems. Lab Chip 15, 1998-2001. doi:10.1039/C5LC00174A Mukhopadhyay, R., 2009. Microfluidics: On the slope of enlightenment. Anal. Chem. 
doi:10.1021/ac900638w

Niño, M.R.R., Patino, J.M.R., 1998. Surface tension of bovine serum albumin and tween 20 at the airaqueous interface. J. Am. Oil Chem. Soc. 75, 1241-1248. doi:10.1007/s11746-998-0169-6

Phurimsak, C., Yildirim, E., Tarn, M.D., Trietsch, S.J., Hankemeier, T., Pamme, N., Vulto, P., 2014. Phaseguide assisted liquid lamination for magnetic particle-based assays. Lab Chip 14, 2334-43. doi:10.1039/c4lc00139g

Poulsen, C.E., Kistrup, K., Andersen, N.K., Taboryski, R., Hansen, M.F., Wolff, A., 2016. Laser ablated micropillar energy directors for ultrasonic welding of microfluidic systems. J. Micromechanics Microengineering 26, 67001. doi:10.1088/0960-1317/26/6/067001

Trietsch, S.J., Rauwé, W., Urban, G. a, Manz, A., Hankemeier, T., Linden, H.J. Van Der, Vulto, P., 2011. the Phaseguide Paradigm : Priming and Emptying of Monolithic Polymer Chips. uTAS 942-944.

Vulto, P., Medoro, G., Altomare, L., Urban, G. a, Tartagni, M., Guerrieri, R., Manaresi, N., 2006. Selective sample recovery of DEP-separated cells and particles by phaseguide-controlled laminar flow. J. Micromechanics Microengineering 16, 1847-1853. doi:10.1088/0960-1317/16/9/013

Vulto, P., Podszun, S., Meyer, P., Hermann, C., Manz, A., Urban, G. a, 2011. Phaseguides: a paradigm shift in microfluidic priming and emptying. Lab Chip 11, 1596-1602. doi:10.1039/c01c00643b

Yildirim, E., Trietsch, S.J., Joore, J., van den Berg, A., Hankemeier, T., Vulto, P., 2014. Phaseguides as tunable passive microvalves for liquid routing in complex microfluidic networks. Lab Chip 14, 3334-40. doi:10.1039/c4lc00261j 